\title{
Antropología
}

\section{Enfoque estructural de las representaciones sociales de los adolescentes sobre el envejecimiento y las personas mayores}

\author{
Structural approach of social representations \\ of teens on aging and elderly
}

\section{Abordagem estrutural das representações sociais de adolescentes sobre envelhecimento e idoso}

Suiany Nascimento Mota ${ }^{1}$, Jéssica de Menezes Nogueira ${ }^{2}$, Bruna Karen Cavalcante Fernandes ${ }^{3}$, Hanna Gadelha Silva ${ }^{4}$, Márcia de Assunção Ferreira ${ }^{5}$, Maria Célia de Freitas ${ }^{6}$

${ }^{1}$ Enfermeira. Universidade Estadual do Ceará. Fortaleza, CE, Brasil.

${ }^{2}$ Enfermeira. Doutoranda em Enfermagem pela Universidade Federal do Rio de Janeiro. Rio e Janeiro, RJ, Brasil. ${ }^{3}$ Enfermeira. Mestranda em Cuidados Clínicos em Enfermagem e Saúde pela Universidade Estadual do Ceará. Fortaleza, CE, Brasil.

${ }^{4}$ Acadêmica de Enfermagem. Universidade Estadual do Ceará. Fortaleza, CE, Brasil.

${ }^{5}$ Enfermeira. Docente da Universidade Federal do Rio de Janeiro, Rio e Janeiro, RJ, Brasil.

${ }^{6}$ Enfermeira. Pós Doutora em Enfermagem. Docente da Universidade Estadual do Ceará. Fortaleza, CE, Brasil.

Cómo citar este artículo en edición digital: Mota, S.N., Nogueira, J.M., Fernandes, B.K.C., Silva, H. G., Ferreira, M.A., Freitas, Ma .

C. (2018). Enfoque estructural de las representaciones sociales de los adolescentes sobre el envejecimiento y las personas mayores.

Cultura de los Cuidados (Edición digital), 22(50). Recuperado de http://dx.doi.org/10.14198/cuid.2018.50.11

Correspondencia: Rua Michele No30 Bairro: Passaré Cidade: Fortaleza/CE. Brasil.

Correo electrónico: suianymota@gmail.com

07/06/2017; Aceptado: 08/11/2017

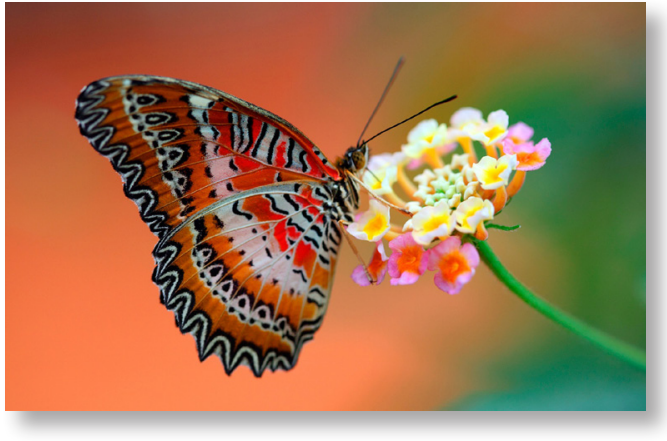

ABSTRACT

Objective: To know the social representations of adolescents on aging and elderly.

Method: A descriptive study supported in Social Representations with the focus on its structural approach, performed in a state school of mid-level and the sample consisted of 99 adolescents. a questionnaire, called
Words of Free Association Test (TALP) using terms like inducing aging and old words was applied. Data analysis was performed using as the tool EVOC 2000 software.

Results: The results of TALP for inducing term "aging" is a list of 422 words, and of these $127(30 \%)$ were different. We note that the word "old" appeared with the largest number of evocations (31), 73 words were mentioned only once. For inducing term "old", the result was a relationship with 418 words in total, and among these terms 151 (36.1\%) were different. We found that the word "care" appears among the most cited with 22 evocations then comes the word "disease" being cited 17 times.

Conclusion: For those teens aging process is strongly directed to the physical aspects as 
“white hair", "wrinkles", “old”, which were very important positions mentioned and words.

Keywords: Social representation, Aging, Old Age, Elderly.

\section{RESUMEN}

Objetivo: Conocer las representaciones sociales de los adolescentes sobre el envejecimiento y los ancianos.

Método: Estudio descriptivo basado en las Representaciones Sociales centrándose en su abordaje estructural, realizado en una escuela provincial secundaria, en el que se incluyó una muestra formada por 99 adolescentes. Se aplicó un cuestionario, llamado Test de Asociación Libre de Palabras (TALP), utilizando como términos inductores las palabras "envejecimiento" y "anciano". El análisis de los datos se realizó utilizando como herramienta el programa informático EVOC 2000.

Resultados: El resultado del TALP para el término inductor "envejecimiento" fue una lista con 422 palabras, donde 127 (30\%) eran diferentes entre sí. Observamos que la palabra "viejo" apareció con el mayor número de evocaciones (31), mientras que 73 palabras fueron evocadas solamente una vez. Para el término inductor “anciano", el resultado fue una relación con 418 palabras en total, de las cuales $151(36,1 \%)$ eran diferentes. Comprobamos que la palabra "atención” aparece entre las más mencionadas, con 22 evocaciones, seguida por la palabra "enfermedades", la cual fue mencionada 17 veces.

Conclusión: Para los adolescentes encuestados, el proceso de envejecimiento está fuertemente orientado hacia los aspectos físicos como "cabellos blancos", "arrugas" y "viejo", que fueron palabras muy evocadas y en posiciones relevantes.

Palabras clave: Representación social, Envejecimiento, Vejez, Anciano.

\section{RESUMO}

Objetivo: Conhecer as representações sociais de adolescentes sobre envelhecimento e idoso.

Método: Estudo descritivo apoiado nas Representações Sociais com o foco em sua abordagem estrutural, realizado em uma escola estadual de nível médio e a amostra foi composta por 99 adolescentes. Foi aplicado um questionário, denominado Teste de Associação Livre de Palavras (TALP), utilizando como termos indutores as palavras envelhecimento e idoso. A análise dos dados foi realizada utilizando como ferramenta o software EVOC 2000.

Resultados: $\mathrm{O}$ resultado do TALP para o termo indutor "envelhecimento", foi uma lista com 422 palavras, sendo que destas 127 (30\%) eram diferentes entre si. Observamos que a palavra "velho" apareceu com o maior número de evocações (31), enquanto 73 palavras foram evocadas apenas uma vez. Para o termo indutor "idoso", o resultado foi uma relação com 418 palavras no total, sendo que dentre esses termos $151(36,1 \%)$ eram diferentes. Verificamos que a palavra "cuidados" aparece entre as mais citadas com 22 evocações, em seguida vem a palavra "doenças" sendo citada 17 vezes.

Conclusão: Para esses adolescentes o processo de envelhecimento está fortemente direcionado para os aspectos físicos como "cabelos brancos", "rugas", "velho", que foram palavras muito evocadas e em posições importantes.

Palavras-chave: Representação social, Envelhecimento, Velhice, Idoso.

\section{INTRODUÇÃO}

O envelhecimento é um processo multidimensional que engloba uma série de fatores e que somente pode ser compreendido levando-se em consideração aspectos biológicos, psíquicos e socioculturais da população (Oliveira, 
2012). Com a predominância do paradigma médico, existe uma hipervalorização das análises que evidenciam, exclusivamente, o aspecto biológico do ser humano. Para este paradigma, o envelhecimento biológico determina mudanças estruturais no corpo do idoso, as quais são percebidas sempre como perdas. Esse discurso termina por naturalizar a ideia da velhice como o momento de "degradação" da condição humana (Cunha, Eulálio \& Brito, 2004). Tal perspectiva constitui-se um equívoco, não só por realçar apenas os aspectos biológicos mais negativos dos sujeitos, mas também por desconsiderar que essas perdas não são determinantes nas mudanças individuais.

Em nossa sociedade, a identidade dos idosos se constrói em função de uma contraposição à identidade do jovem, opondo-se às qualidades de atividade, força, memória, beleza, potência e produtividade, sendo o envelhecimento pouco aceito ou trabalhado pelas pessoas, direcionando a relação cultural entre velhice e morte, com o predomínio do culto à juventude. Desta forma, percebemos que o envelhecimento é visto de maneira preconceituosa e estereotipada por muitas pessoas, como uma fase do desenvolvimento humano que é marcado por acontecimentos negativos, mesmo sendo cada vez mais evidente a participação ativa dos idosos em nosso meio. (Cótica, 2011; Teixeira et al., 2012).

A velhice é uma categoria construída socialmente, assim como a adolescência e a juventude. É vista e tratada de maneira diferente, de acordo com períodos históricos e com as estruturas sociais, culturais, econômicas e políticas de cada povo. Destarte, não se pode ignorar que há uma herança cultural e científica que veicula mensagens negativas acerca do envelhecimento em geral e do idoso em particular (Matos \& Neves, 2013).

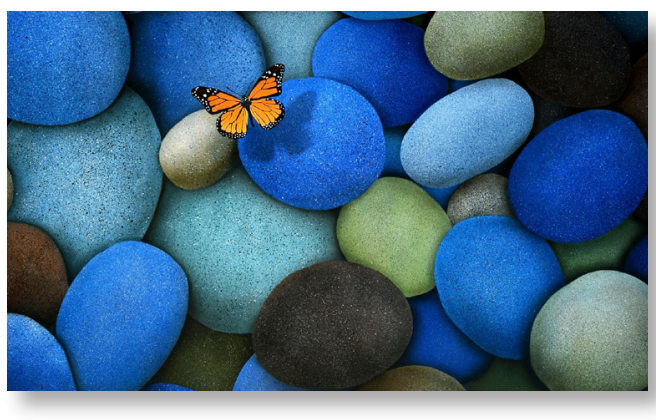

Muitas vezes, o idoso é visto pelos mais jovens como alguém sem expectativas de vida, sem maiores oportunidades na sociedade e que acaba sendo alienado ao que lhe é proporcionado, sendo muitas vezes ligado à visão de incapacidade física e doença (Gvozd \& Dellaroza, 2012). A compreensão do envelhecimento deve ir além de uma abordagem multidimensional que forneça uma visão global da situação social e de saúde da pessoa idosa. Faz-se necessário considerar as representações que cada indivíduo possui dos fatores que lhes são pertinentes, ou seja, que é ser idoso e em que consistem seus papéis sociais; que significa autonomia e que dificuldades afetam; representações de saúde; qualidade de vida, entre outros (Ribeiro et al., 2002; Alves, Eulálio \& Brito, 2004). Desse modo, a teoria das representações sociais apresenta-se como uma das alternativas dessa nova perspectiva de compreensão do processo saúde-doença, uma vez que essa teoria busca apreender, ao mesmo tempo, a dimensão cognitiva e a social desse fenômeno. Nessa perspectiva, objetivou-se conhecer as representações sociais de adolescentes sobre envelhecimento e idoso.

\section{MATERIAIS E MÉTODOS}

Trata-se de um estudo descritivo, de natureza qualitativa, apoiado nas Representações Sociais (RS) com o foco em sua abordagem estrutural que enfatiza os conteúdos cognitivos das representações, com o objetivo de identi- 
ficar sua estrutura e organização. As representações sociais são consideradas um sistema sócio-cognitivo que pode ser ao mesmo tempo rígido e flexível, estável e móvel. Para contemplar estas características aparentemente contraditórias, a abordagem estrutural propõe que uma representação social é formada por dois sistemas distintos, mas complementares o central e o periférico (Sá, 2002).

O cenário do estudo foi uma escola estadual de nível médio na cidade de Fortaleza-Ce. A população do estudo foi constituída por 351 alunos, de ambos os sexos, matriculados na referida instituição de ensino. A amostra foi composta pelos alunos matriculados no oitavo ano, considerando que essas turmas apresentavam disponibilidade de horário nos dias definidos para a coleta dos dados. Foram adotados os seguintes critérios de inclusão: estar devidamente matriculado na escola e ter idade entre 12 a 18 anos completos, idade que a pessoa é considerada adolescente segundo o Estatuto da Criança e do Adolescente. Foram excluídos da pesquisa os indivíduos que apresentaram algum tipo de déficit cognitivo que comprometiam sua autonomia, totalizando 99 adolescentes que participaram do estudo.

A pesquisa foi realizada nos meses de março a maio de 2015. Foi aplicado um questionário, denominado Teste de Associação Livre de Palavras (TALP), utilizando como termos indutores as palavras envelhecimento e idoso. Utilizou-se a técnica das evocações das palavras e a análise dos dados foi realizada utilizando como ferramenta o software EVOC 2000. O EVOC 2000 auxiliou na construção de um quadro de quatro quadrantes que podem ser interpretados da seguinte maneira: no primeiro quadrante situam-se os elementos de maior importância e, por isso, possíveis de constituírem o núcleo central de uma repre- sentação. Estes elementos são os mais evocados e citados com maior frequência pelos sujeitos, e mais resistente a mudanças. $\mathrm{O}$ segundo e o terceiro quadrantes correspondem aos elementos menos salientes na estrutura da representação, contudo eles são significativos em sua organização. No segundo quadrante estão os elementos que obtiveram uma frequência alta, mas que foram citados em últimas posições; no terceiro quadrante, zona de contraste, encontram-se os elementos que foram citados numa frequência baixa, porém foram evocados primeiramente. No quarto quadrante estão os elementos que correspondem à segunda periferia (Vergés, 2002).

O presente estudo foi aprovado pelo Comitê de Ética e Pesquisa da Escola de Enfermagem Anna Nery com parecer favorável $n^{\circ}$ 856.631e seguiu todas as recomendações éticas da pesquisa com seres humanos regulamentados pela Resolução 466 de 2012 do Conselho Nacional de Saúde. (Brasil, 2012)

\section{RESULTADOS E DISCUSSÕES}

Constatou-se que $55 \%$ da amostra era do gênero feminino e $45 \%$ do masculino. A idade dos sujeitos pesquisados está compreendida entre 13 a 18 anos, sendo que $34 \%$ tinham 15 anos, 29\% tinham 14 anos, 13\% tinham 13 anos, $12 \%$ tinham 16 anos, e os demais (11\%) tinham entre 17 e 18 anos de idade. No estudo, predominaram os alunos do $8^{\circ}$ ano do ensino fundamental com $77 \%$ do total, e os demais $(22 \%)$ eram do $1^{\circ}$ ano do ensino médio.

$O$ resultado do TALP para o termo indutor "envelhecimento", foi uma lista com 422 palavras, sendo que destas 127 (30\%) eram diferentes entre si. Observamos que a palavra "velho" apareceu com o maior número de evocações (31), enquanto 73 palavras foram evocadas apenas uma só vez. 
Quadro 1 - Elementos centrais e periféricos da RS sobre envelhecimento para adolescentes escolares com o termo indutor "envelhecimento". Fortaleza, 2016.
Os elementos centrais expostos no quadro 1 trazem o significado do idoso voltado para aspectos físicos, velho (31) e rugas (21), sendo essas as palavras demaior frequência. A palavra experiência (20) também foi bastante lembrada pelos adolescentes quando se falava em envelhecimento, isso se deve a todo conhecimento adquirido por essas pessoas, ao longo da vida, através de suas vivencias. Além disso, para os jovens, o envelhecimento também representa uma necessidade de cuidados (12), provavelmente por conta das alterações inerentes ao processo de envelhecimento.

No quadro 1, oferecida pelo EVOC, ficam distribuídos os dados das ocorrências em quatro quadrantes, que nos permitem visualizar o núcleo central, de contraste e periféricos de uma representação. No primeiro quadrante estão os elementos centrais: "Cabelos brancos", "cuidados", "experiências", "idade", “idoso", “rugas" e "velho".Já os elementosperiféricos da representação social de adolescentes sobre o envelhecimento, encontram-se distribuídosnos três demais quadrantes: no superior direito, as palavras "avos", “doenças" e "tempo" pertencem à primeira periferia; no quadrante inferior esquerdoestão os termos "fraqueza", "idade avançada", "maturidade", "respeito" e "tristeza" que constituem a zona de contraste, e, no quadrante inferior direito, segunda periferia,estão localizadas as palavras "amadurecimento", "aposentadoria", "cansaço", "carinho", "chato", "histórias", "morte", "velhice" e "vida". $2^{a}$ periferia

$\begin{array}{cc}6 & 2,833 \\ 5 & 3,400 \\ & \\ 8 & 2,750 \\ 5 & 3,200 \\ 9 & 3,333 \\ 6 & 3,667 \\ 8 & 3,500 \\ & \\ 9 & 2,889 \\ 6 & 3.167\end{array}$

ante inferior direito

\section{mento.}

Percebemos que o núcleo central dos adolescentes aponta o envelhecimento fortemente marcado por características físicas, identificado pelas palavras "cabelos brancos", "idade", "idoso", "rugas" e "velho". No entanto, na periterísticas físicas as pessoas que passa por esse processo podem ser geradores de fraqueza, maturidade, mas que também exigem respeito.

O processo de envelhecimento provoca no organismo modificações biológicas, psicológicas e sociais; porém, como já referido, é na velhice que este processo aparece de forma mais evidente. As modificações biológicas são as morfológicas, reveladas por aparecimento de rugas, cabelos brancos, o que fazem relação direta com o senso comum desses adolescentes sobre as RS de envelhecimento (Santos, 2010). É inegável que os adolescentes se preocupam com a boa aparência e relaciona o envelheciferia, os jovens apontam que com essas carac- 
mento com a presença de rugas, cabelo branco, principalmente na sociedade atual em que se cultua a beleza, o novo, e a pessoa idosa é sempre vista em oposição ao jovem e ao belo. As rugas, na velhice, parecem o resultado do tempo vivido, mas também é preciso se levar em conta o fator hereditariedade e o organismo individual. Durante o envelhecimento, há alterações elásticas nas fibras da pele e dos órgãos que se transformam em sua composição e determinam as mudanças nas características pessoais (Carvalho Filho, 2007).

Segundo Cruz e Ferreira (2011) ser jovem é ser forte. Ser velho é ser fraco, sem forças para lutar. Não só o velho se sente assim, mas também a sociedade lhe reforça a ideia, quase que diariamente, principalmente através dos meios de comunicação, de que bonito é o jovem, que é forte. $\mathrm{E}$, diretamente relacionada a essa questão de forte versus fraco, identifica-se a associação da velhice com a doença e, como consequência, a velhice com a necessidade de cuidados, questão que ficou clara na pesquisa uma vez que a palavra "cuidado" foi lembrada 12 vezes pelos adolescentes.

Desta forma, ficou claro que os adolescentes veem o processo de envelhecimento como algo ruim, que traz consigo alterações físicas e biológicas, e que com o tempo as

\begin{tabular}{|c|c|c|c|c|c|c|}
\hline & Rang $<2,7$ & & & Rang $>2,7$ & & \\
\hline & Termo Evocado & $\begin{array}{l}\text { Fre } \\
\mathrm{g}\end{array}$ & OME & Termo Evocado & Freq & OME \\
\hline \multirow[t]{5}{*}{ Freq Média } & \multicolumn{3}{|c|}{$\begin{array}{c}\text { Quadrante superior esquerdo } \\
\text { Núcleo Central }\end{array}$} & \multicolumn{3}{|c|}{$\begin{array}{c}\text { Quadrante Superior Direito } \\
1^{\mathrm{a}} \text { Periferia }\end{array}$} \\
\hline & Amor & 9 & 2,556 & Aposentadoria & 17 & 3,294 \\
\hline & Avos & 18 & 1,722 & Cabelos brancos & 10 & 2,700 \\
\hline & bengala & 9 & 2,667 & Doenças & 17 & 3,059 \\
\hline & Chato & 11 & 2,636 & & & \\
\hline \multirow{2}{*}{$>=9$} & Cuidados & 22 & 2,455 & & & \\
\hline & Experiencias & 14 & 2,500 & & & \\
\hline
\end{tabular}

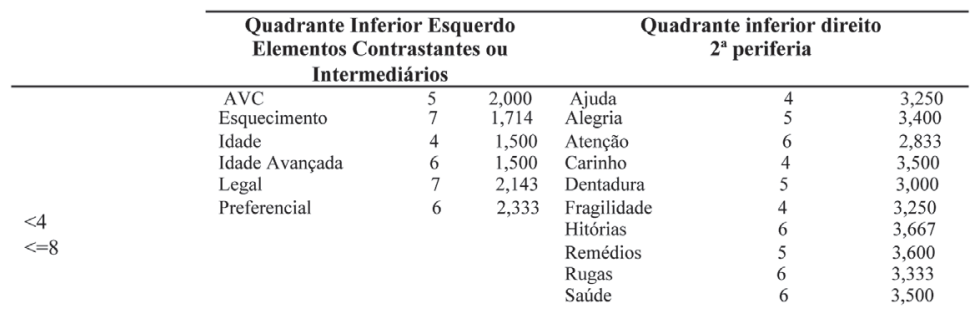

em que vivemos numa sociedade que se cultua a beleza, o aparecimento de cabelos brancos, de rugas, embora seja um indicativo natural do envelhecer, é sinalizado cultural e socialmente como algo negativo.

A pesquisa também procurou saber como os adolescentes escolares representavam a pessoa idosa. O quadro 2, também oferecida pelo EVOC, mostra o resultado do TALP para a indução "idoso". O resultado foi uma relação com 418 palavras no total, sendo que dentre esses termos 151 (36,1\%) eram diferentes. Verificamos que a palavra "cuidados" aparece entre as mais citadas com 22 evocações, em seguida vem a palavra "doenças" sendo citada

Quadro 2 - Elementos centrais e periféricos da RS sobre envelhecimento para adolescentes $z a, 2016$. 17 vezes. escolares com o termo indutor "idoso". Fortalepessoas que passam por esse processo ficam fracos, cansados, doentes e tristes, mas se tornam maduros e merecem respeito. Essas características reforçam ainda mais a figura estereotipada do envelhecimento como enfatizam Almeida e Lourenço (2009) 
rior direito estão situados os termos que constituem a primeira periferia: "aposentadoria", “cabelos brancos", “doenças” e "respeito”. Já no quadrante inferior esquerdo estão as palavras: "AVC", "esquecimento", "idade", "idade avançada", "legal" e "preferencial", que compõem a zona de contraste. $\mathrm{O}$ quadrante inferior direito, a segunda periferia, é composto pelas palavras: “ajuda”, “alegria”, "atenção", “carinho”, "dentadura”, "fragilidade", "histórias", "remédios", "rugas" e "saúde".

Conforme os elementos centrais expostos no quadro 2, percebemos que os léxicos que compõem os saberes sobre idoso, assim como o de envelhecimento, é bastante marcado por aspectos físicos, com as palavras velho (9) e bengala (9), porém sua essencialidade está voltada para os elementos afetivos identificadas nas palavras "amor", "avos" (palavra que faz uma ligação direta como os sentimentos que os avos representam, como carinho, amor, respeito, atenção) que embora não tenham sido as palavras de maior evocação, mas as vezes que foram ditas foram as primeiras a serem lembradas, vindo sempre em posições significantes como $1^{\circ}$ e $2^{\circ}$ lugar.

A palavra "avós", evocada 18 vezes entre a primeira e segunda posição, representa o pensamento de adolescentes que fazem uma relação direta entre a pessoa idosa a imagem física, psicológica e social de seus avós, que pode ter significados ora positivos, quando forem avós ativos, independentes, saudáveis, e ora negativos, quando forem avós doentes, debilitados, que necessitam de cuidados.

Os resultados apontam que os resultados dos núcleos centrais, tanto do termo "envelhecimento" quanto da palavra "idoso" remete a uma representação. Segundo Dias e Silva (2003) os avós são vistos como segundos pais e como pessoas que exercem influência na vida dos netos, tanto no aspecto pessoal como no social de seus netos. Isso justifica o pensamento de adolescentes ao relacionarem "idoso" às palavras "amor", "sabedoria", "historias".

As interações entre idosos com outras gerações, leva a uma vivência das relações intergeracionais com base em atividades de compartilhamento que promovem a interação social (entretenimento, refeições, conversas) onde mobiliza na família, incluindo-se os adolescentes, sentimentos: como ser amor, compreensão e apoio. (Matos \& Neves, 2013).

Isto é um traço que caracteriza a RS, pois ao se depararem com a palavra "idoso" os adolescentes, trazem como núcleo central palavras que trazem a mobilização de sentimentos, afetos e julgamentos, como podemos constatar pelos léxicos "amor", "chato" e "avós". Além disso, a aproximação entre jovens e a pessoa idosa poderão proporcionar trocas intergeracionais podem beneficiar o idoso ao permitir que este utilize sua experiência de vida acumulada, transmitindo o passado, sua cultura, seus valores, sua história de vida. Assim, os adolescentes podem construir uma concepção positiva da velhice, fortalecendo seu relacionamento com os idosos e transmitindo-lhes sua vitalidade e alegria (Mazuttie \& Scortegagna, 2006). Para Cruz e Ferreira (2011) dizem que ao envelhecer e se tornar fraco, o velho se torna susceptível a doença. Por isso a correlação da velhice com a doença, se não existe velho forte, consequentemente ele será doente porque está fraco, sem forças para lutar. Só luta quem é forte, e essa é uma qualidade que o velho não possui.

Nos dois quadros, observa-se a predominância por evocações relacionadas a condições biológicas em que o adolescente associa o processo de envelhecimento e a pessoa idosa à aspectos degradantes, e que são vistos a partir 
das alterações comuns ao processo de envelhecimento, principalmente no que diz respeito ao declínio funcional e cognitivo.

Quando a velhice é considerada como boa, ela carrega consigo a ideia de trazer sabedoria e experiência que podem ser compartilhadas com os mais jovens. A experiência está relacionada com maior tempo de vida, então os mais velhos possuem esta vantagem por já terem passado por várias situações e saberem como agir, de forma mais sábia, em determinadas ocasiões. Sabedoria e experiência fazem parte da vida de todo mundo, pois, à medida que vamos vivendo e envelhecendo, vamos adquirindo-as, de acordo com as escolhas, acertos, erros, relações, desamores, relações interpessoais, amores, perdas, ganhos, viagens, trabalhos, entre outras situações (Oliveira, 2009).

Segundo Mazutti e Scortegagna (2006) a experiência de vida transmite-se por meio da convivência, das trocas que acontecem no dia-a-dia nas relações que se estabelecem no contexto familiar. Essa interação pode ser benéfica tanto para os adolescentes como para os idosos, conforme são construídos os vínculos afetivos. A partir dos dados da pesquisa, fica evidente que os adolescentes ainda não despertaram para as oportunidades que a velhice pode trazer, fazendo com que a compreensão daqueles acerca do envelhecimento se resuma apenas a rugas, cabelos brancos, doenças, cuidados e morte. Desta forma, é importante que os jovens também sejam estimulados e orientados sobre o envelhecimento saudável e ativo. Rocha-Coutinho (2006) destaca que hoje, entende-se que a velhice, assim como a infância, a adolescência e a maturidade, mais do que simples fases da vida, são categorias socialmente construídas, que só alcançam seu sentido através do discurso. Ser velho, da mes- ma forma que ser criança, jovem ou adulto, está associado aos valores vigentes numa dada sociedade em um determinado tempo.

\section{CONCLUSÃO}

No estudo foi possível identificar diversos elementos que compõem as percepções dos adolescentes escolares acerca do envelhecimento e do idoso. Para esses jovens o processo de envelhecimento está fortemente direcionado para os aspectos físicos como "cabelos brancos", "rugas", "velho", que foram palavras muito evocadas e em posições importantes. No entanto, para o ser humano idoso, os adolescentes trazem elementos sociais, psicológicos e afetivos.

Além disso, percebeu-se grande relação entre a figura que o idoso representa com os avós dos adolescentes, o que mobiliza amor, afeto e cuidados, o que se constata que o relacionamento intergeracional é um fator importante para a construção da RS da população sobre o que é ser idoso e quais implicações têm para as relações humanas no que diz respeito à sociedade envelhecente.

Ademais, os resultados apontaram que as percepções que cabem na estrutura do pensamento dos adolescentes sobre o envelhecimento e a pessoa idosa, na grande maioria das vezes, se dão por meio de uma ideia negativa sobre o processo. Observamos, também, que para os jovens, a velhice não é determinada apenas pelo tempo, critério cronológico, mas principalmente pelos fatores biológicos e psicológicos. Nesse contexto, mostra-se necessário a educação gerontológica como ferramenta de apoio para a preparação da sociedade, principalmente dos jovens, para o processo de envelhecimento além de mobilizar um novo olhar e pensamento para a imagem do idoso no imaginário social. 


\section{REFERÊNCIAS}

- Almeida, T., \& Lourenço, M. (2009). Reflexões: conceitos, estereótipos e mitos acerca da velhice. Revista Brasileira de Ciências do Envelhecimento Humano, 6(2), 233-244. Recuperado de http://www.upf.br/seer/index.php/ $\underline{\mathrm{rbceh} / \mathrm{article} / \mathrm{view} / 171}$

- Alves, R. F., Eulálio, M. C., \& Brito, S.M.O. (2004). Representações sociais: via de acesso ao pensamento social sobre a saúde-doença. In: Fernandes, A., Carvalho, M. R., Sobrinho, M. D. (Org.) Representações sociais e saúde: construindo novos diálogos, 1. ed., Campina Grande: EDUEP.

- Brasil. (2012). Ministério da Saúde. Conselho Nacional de Saúde. Resolução nº 466 de 12 de dezembro de 2012. Diretrizes e normas regulamentadoras de pesquisas envolvendo seres humanos. Brasília-DF.

- Carvalho Filho, E. T. (2007). Fisiologia do envelhecimento. In: Papaléo Netto, M. Tratado de Gerontologia. 2 ed. São Paulo: Atheneu.

- Cótica, C. S. (2011). Percepção de Envelhecimento e Finitude no Final da Vida Adulta Tardia. Geriatria \& Gerontologia, 5(4), 201-213. Recuperado de http://repositorio.unb.br/handle/10482/8826

- Cruz, R.C., \& Ferreira, M.A. (2011). Um certo jeito de ser velho: representações sociais da velhice. Texto Contexto Enferm. Florianópolis, 20(1), 144-151. Recuperado de http://dx.doi.org/10.1590/S0104-07072011000100017

- Cunha, A. C. H., Eulálio, M. C., \& Brito, S. M. O. (2004). O corpo e suas representações construídas por mulheres idosas. In: Fernandes, A., Carvalho, M.R., Domingos Sobrinho, M. Representações Sociais e Saúde: construindo novos diálogos, 1. ed., Campina Grande: EDUEP.

- Dias, C. M. S. B, \& Silva, M.A.S. (2003). Os avós na perspectiva de jovens universitários. Psicologia em Estudo. Maringá, 8 (esp), 55-62. Recuperado de https://dx.doi. org/10.1590/S1413-73722003000300008

- Gvozd, R., \& Dellaroza, M. S. G. (2012). Velhice e a Relação com Idosos: O Olhar de Adolescentes do Ensino Fundamental. Rev. Bras. Geriatr. Gerontol. Rio de Janeiro, 15(2), 295-304. Recuperado de http://dx.doi. org/10.1590/S1809-98232012000200012

- Matos, A. D., \& Neves, R. B. (2013). Les nouvelles rela- tions intergénérationnelles des personnes âgées en famille d'accueil. Une expérience portugaise. Portugal: Retraite et société.

- Mazutti, C, \& Scortegagna, H. M. (2006). Velhice e envelhecimento humano: concepções de pré-escolares do município de Tapejara - RS. Revista Brasileira de Ciências do Envelhecimento Humano, 3(2), 101-112. Recuperado de http://www.upf.br/seer/index.php/rbceh/ article/view/77

- Oliveira, A. M.M, Lopes, M. E. L., Evangelista, C. B., Oliveira, A. E. C., Gouvéia, A. M. L., \& Duarte, M. C. S. (2012). Representações Sociais e Envelhecimento: uma Revisão Integrativa de Literatura. Revista Brasileira de Ciências da Saúde, 16(3), 427-434. Recuperado de http://periodicos.ufpb.br/ojs/index.php/rbcs/article/ view/12801

- Oliveira, S. A. P. (2009). O jovem frente à velhice e ao envelhecimento. Estudo realizado com alunos de 15 a 18 anos de escola pública na região do Itaim Paulista, São Paulo. Dissertação (Mestrado em Gerontologia), Pontifícia Universidade Católica de São Paulo: São Paulo.

- Ribeiro, R. C. L., Silva, A.I.O., Modena, C.A., \& Fonseca, M.C. (2002). Capacidade funcional e qualidade de vida de idosos. Estud. interdiscipl. envelhec., 4(1), 85-96. Recuperado de http://www.seer.ufrgs.br/RevEnvelhecer/ article/view/4721

- Rocha-Coutinho, M. L. (2006). Transmissão geracional e família na contemporaneidade. In: Barros, M. M. L (Org.). Família e gerações. Rio de Janeiro: FGV.

- Sá, C.P. (2002). Núcleo central das representações sociais. Revista. Petrópolis: Vozes.

- Santos, S. S. C. (2010). Concepções teórico-filosóficas sobre envelhecimento, velhice, idoso e enfermagem gerontogeriátrica. Rev. bras. enferm., Brasília, 63(6), 10351039. Recuperado de http://dx.doi.org/10.1590/S003471672010000600025

- Teixeira, M. E. C. et al. (2012). Envelhecimento e Percepção corporal de idosos institucionalizados. Rev. Bras. Geriat. Geront. Rio de Janeiro, 15(1), 63-68. Recuperado de http://dx.doi.org/10.1590/S1809-98232012000100007

- Vèrges, P. (2002). Conjunto de programas que permitem a análise de evocações: EVOC: manual. Versão 5. 